\title{
Attachment Style and Resiliency in Patients with Obsessive-Compulsive Personality Disorder
}

\author{
Ali Zakiei', Mostafa Alikhani ${ }^{2, *}$, Vahid Farnia², Zinab Khkian³, Jalal Shakeri², Sanobar Golshani ${ }^{2}$ \\ 'Social Development and Health Promotion Research Center, Kermanshah University of Medical Sciences, Kermanshah, Iran \\ ${ }^{2}$ Department of Psychiatry, Substance Abuse Prevention Research Center, Kermanshah University of Medical Sciences, Kermanshah, Iran \\ ${ }^{3}$ Department of Psychology, Faculty of Social Sciences, Razi University, Kermanshah, Iran
}

Background: The goal of the present study was to determine the relationships between attachment styles and resiliency in obsessive-compulsive personality disorder.

Methods: A random sample of 260 subjects was obtained from the population of undergraduate students of the Nour Branch of Islamic Azad University, which is located in Mazandaran, and these subjects were enrolled in this descriptive and correlational study. The collected data included the subjects' responses to an adult attachment style questionnaire, resilience scale, and obsessive-compulsive personality disorder questionnaire. The data were analyzed with Pearson correlation coefficient indices and multiple regressions.

Results: The results of the data analysis showed a positive correlation (relationship) between ambivalent/avoidant attachment styles and obsessive-compulsive personality disorder and a negative correlation between resilience and obsessive-compulsive personality disorder. Furthermore, these results demonstrated that attachment style and resiliency can predict obsessive-compulsive personality disorder. In addition, no significant relationships were found between the demographic variables (convertibles) and obsessive-compulsive personality disorder. Conclusion: These results suggested that attachment style and resiliency contribute to the development of obsessive-compulsive personality disorder.

Keywords: Compulsive Personality Disorder; Object Attachment; Psychological Resilience; Students 


\section{INTRODUCTION}

Obsessive-compulsive personality disorder is a multilateral behavioral-thought pattern involving preoccupations with orderliness, perfectionism, and mental and intrapersonal control that results in a loss of flexibility, openness, and efficiency. ${ }^{1)}$ A number of studies have shown that parents with certain characteristics, such as excessive child supportiveness, high expectations, strictness, and oversensitivity, tend to have children who possess a high level of obsessive-compulsive symptoms and anxious thoughts. ${ }^{2)}$

Bowlby, ${ }^{3)}$ an attachment theorist, has suggested that humans are born with an innate and adaptable motivational system that is designed with the need to seek proximal figures of attachment, especially in response to threats, to enhance security. During frequent interactions with a supportive and sensitive caregiver, a child develops a stable emotional-cognitive structure that reduces stress and provides peace, comfort, and protection from threatening situations. ${ }^{4)} \mathrm{Re}$ searchers believe that parents treat their children differently based on their personality type and psychological traits. The diverse training and behavioral methods of parents results in three attachment styles: secure, avoidant insecure, and ambivalent insecure. ${ }^{5)}$ Bowlby ${ }^{6}$ in 1969 has speculated that many personality disorders are derived from and affected by the deprivation of a child of the care and attention of a caregiver or lack of stability in the relationship of the child with the person to whom he/she is attached. ${ }^{6)}$ The results of the studies of Mikulincer ${ }^{7)}$ have demonstrated that people with insecure attachment styles are less able to cope with stressful situations.

In addition to the attachment styles that are related to the traditional structure, a new structure has recently caught the attention of psychologists. A number of studies have described factors that moderate the effects of stressful events that contribute to the development of psychological disorders and that protect individuals from these disorders. One factor that allows people to better manage the needs, challenges, and threats of life is resiliency. ${ }^{8)}$ Resilient individuals exhibit more adaptive behaviors in response to negative life events. ${ }^{9)}$

Studies by Bonanno ${ }^{10)}$ in 2004, Masten ${ }^{11)}$ in 2001, Connor and Davidson $^{12)}$ in 2003, and Basu ${ }^{13)}$ in 2004 have shown that individuals with less resiliency for life events experience mental pressure, anxiety, and/ or depression. Friborg et al. ${ }^{14)}$ have suggested that resilient people are more flexible against traumatic situations and are better able to protect themselves against these conditions.

However, it is unclear if resiliency is associated with obsessive-compulsive personality disorder and whether attachment style and resiliency can together predict obsessive-compulsive personality disorder. Because more research is needed on obsessive-compulsive personality disorder and the roles of attachment style and resiliency in predicting this disorder; the objective of the present study was to determine if obsessive-compulsive personality disorder is related to attachment style and resiliency among students.

\section{METHODS}

This descriptive/correlational study was designed to investigate the relationship between variables that might play a role in obsessive-compulsive personality disorder. The sampling population consisted of all of the undergraduate students of the Nour Branch of Islamic Azad University in Mazandaran. A Morgan table was used to select a sample size of 300 subjects according to the number of research variables. The 300 subjects were selected with a random sampling method that involved the assignment of a number to each student's name. Numbers were then randomly selected, and the students with the corresponding number were included in the study sample. Forty subjects were excluded from the study because of damaged questionnaires. The data for 260 subjects were analyzed in the study. After the research protocol was approved by the Ethics Committee of the Psychodynamic Research Center (Razi Psychology Center), the registration code of 94.120 was used, and the questionnaires were distributed to the students who consented to participating in the study after they were assured of the confidentiality of their shared information. The age range of the sampled students was 19-26 years old, and they had no histories of chronic physical or mental illness. In addition, the subjects were celibate and living with their parents. The data were analyzed with descriptive statistics, Pearson correlation coefficients, and multiple regressions. In addition, a multivariate regression analysis that adjusted for resilience was performed. The three questionnaires described below were used to collect data.

\section{Questionnaire of Adult Attachment Style}

This questionnaire, which was created by Hazan and Shaver ${ }^{15)}$ in 1987, is a self-reporting tool that was designed based on Ainsworth's triple attachment styles (secure, avoidant, and ambivalent). This scale consists of descriptions of the individual's perceptions of his/her relationships with important people in his/her current life. ${ }^{15)}$ Taheri ${ }^{16)}$ in 2010 calculated that the internal consistency of this attachment style questionnaire was 0.55 to 0.77 and the reliability was 0.84 for secure attachment and 0.78 for insecure, ambivalent, and avoidant attachments.

\section{Resilience Questionnaire}

This questionnaire was used to measure resiliency according to Connor and Davidson's resilience scale, which consisted of 25 items and which was designed to measure a subject's ability to deal with pressure and stress. ${ }^{12)}$ This scale assesses various aspects of resiliency, such as feelings of personal ability, resistance to negative events, positive acceptance of change, trust in one's instincts, sense of support and security, spiritual faith, and pragmatic approaches to problem solving. The initial studies of this tool have shown that it is suitable for measuring the resilience of adults. ${ }^{12)}$ In Iran, Jowkar ${ }^{17}$ in 2007 reported an internal consistency for this scale of 0.80 with Cronbach's alpha method. In this scale, a five-option calibration range is implemented for each item (from absolutely false to always true). The minimum tribal resilience score for this scale is zero, and the maximum score is $100 .{ }^{12)}$ 


\section{Obsessive-Compulsive Personality Questionnaire}

In order to assess obsessive-compulsive personality disorder, the obsessive-compulsive personality questionnaire that was designed by Brannigan ${ }^{18)}$ was used. The questionnaire that we used in the current study was derived from a book by Brannigan ${ }^{18)}$ that was translated into Persian by Faride Alagha in 2003. Because the validity and reliability of this questionnaire have not been determined for Iranian subjects, the questionnaire was subjected to exploratory and confirmatory factor analyses. The results of the analysis yielded a Cronbach's alpha value of 0.72 , which indicated its validity. This questionnaire included 22 items that were in the form of yes/no questions. 'Yes' answers yielded 1 point, and 'no' answers resulted in 0 points. Higher scores indicated more symptoms of obsessive-compulsive personality disorder. Only one factor was obtained in the exploratory factor analysis, and these results were confirmed in the confirmatory factor analysis. Exploratory factor analyses were done on the questionnaires, and the Kaiser-Meyer-Olkin value was calculated as 0.867 . Bartlett's test of sphericity equaled 2,030.43 which was significant $(\mathrm{P}<0.001)$ and which justified the factor analysis according to the correlation matrix.

\section{RESULTS}

Of the 260 samples, 186 (71.5\%) were female, and 74 (28.5\%) were male. Seventy-seven subjects were 19-21 years old (29.6\%), 134 were between 22 and 24 years old (51.5\%), and 49 were $25-27$ years old (18.8\%). Twenty-three were married (8.8\%), and 237 were single

Table 1. Baseline characteristics of the study subjects $(\mathrm{N}=260)$

\begin{tabular}{lc}
\hline \multicolumn{1}{c}{ Variable } & Value \\
\hline Demographics & \\
Gender & \\
Female & $186(71.5)$ \\
Male & $74(28.5)$ \\
Age $(y)$ & \\
$19-21$ & $77(29.6)$ \\
$22-24$ & $134(51.5)$ \\
$25-27$ & $49(18.8)$ \\
Marital status & \\
Married & $23(8.8)$ \\
Single & $237(91.2)$ \\
Economic situation & \\
Poor & $40(15.4)$ \\
Medium & $157(64.2)$ \\
Good & $63(20.4)$ \\
Family history of mental disorder & \\
Yes & $11(4.23)$ \\
No & $249(95.77)$ \\
Attachment style & \\
Secure & \\
Ambivalent & $21.14 \pm 17.4$ \\
Avoidant & $36.12 \pm 96.4$ \\
Resiliency & $85.12 \pm 55.5$ \\
Obsessive-compulsive personality disorder & $95.80 \pm 25.12$ \\
\hline
\end{tabular}

Values are presented as number (\%) or mean \pm standard deviation.
(91.2\%). The economic conditions of the subjects were poor $(n=40$, $15.4 \%)$, average $(n=157,64.2 \%)$, or good $(n=63,20.4 \%)$. Eleven of the 260 subjects $(4.23 \%)$ had a family history of mental illness. The mean age of the subjects was 22.61 years old, and their mean scores for secure attachment style, ambivalent attachment style, avoidant attachment style, resiliency, and obsessive-compulsive personality disorder were $14.21,12.36,12.85,80.95$, and 6.31 , respectively. The means and standard deviations of all of the variables are presented in Table 1. Considering the normal distribution of the variables, correlation coefficient were administered to examine the relationship between attachment style and resiliency with obsessive-compulsive personality disorder. The Pearson correlation coefficients for the relationships between ambivalent attachment style and avoidant attachment style with obsessive-compulsive personality disorder were 0.22 and 0.36 , respectively $(\mathrm{P}<0.001$, for both). In contrast, the demographic variables and secure attachment style did not exhibit relationships with obsessivecompulsive personality disorder. A significant correlation coefficient of $-0.23(\mathrm{P}<0.001)$ was achieved between resiliency and obsessive-compulsive personality disorder. The results of the correlation coefficient calculations between the attachment styles/resiliency with obsessivecompulsive personality disorder are presented in Table 2 . We used a multiple regression analysis to investigate the effects of attachment style and resiliency on obsessive-compulsive personality disorder. The results of the regression analysis, which are shown in Table 3, showed that the regression model was meaningful. In the model, the dependent variable was obsessive-compulsive personality disorder, and the independent variables were resiliency and attachment styles. The variance inflation factor, tolerance, and Durbin-Watson results revealed that the regression analysis met the assumption of no collinearity. The results showed that $17 \%$ of the variance related to obsessive-compulsive personality disorder was explained by attachment style and resiliency. The effect size of the avoidant attachment style was $30 \%$ $(\mathrm{P}<0.001)$, while the effect size of the ambivalent attachment style was not significantly meaningful. In addition, the effect size of resiliency on obsessive-compulsive personality disorder was $-0.19(\mathrm{P}<0.001)$. The results of the resiliency-adjusted regression analysis showed a significant relationship $(\mathrm{P}<0.001)$ between the avoidant attachment style

Table 2. Correlation coefficients for the relationships between the demographic variables, attachment styles, resiliency, and obsessive-compulsive personality disorder

\begin{tabular}{lcc}
\hline \multicolumn{1}{c}{ Variable } & Pearson's R & P-value* $^{*}$ \\
\hline Gender & 0.09 & 0.11 \\
Age $(\mathrm{y})$ & 0.02 & 0.68 \\
Marital status & 0.009 & 0.89 \\
Economic situation & 0.008 & 0.71 \\
Family history of mental disorder & 0.08 & 0.10 \\
Secure style & -0.01 & 0.77 \\
Ambivalent style & 0.22 & $<0.001$ \\
Avoidant style & 0.36 & $<0.001$ \\
Resiliency & -0.23 & $<0.001$ \\
\hline
\end{tabular}

*Obtained from the Pearson correlation coefficients. 
Table 3. Results of the regression analysis of the effects of attachment style and resiliency on obsessive-compulsive personality disorder

\begin{tabular}{lccccccccccc}
\hline \multicolumn{1}{c}{ Variable } & $\mathrm{R}$ & $\mathrm{R}^{2 *}$ & $\mathrm{~F}$ & $\mathrm{P}_{\text {-value }}^{\dagger}$ & $\mathrm{B}^{\ddagger}$ & $\mathrm{B}^{\S}$ & $\mathrm{T}$ & P-value $^{\text {Il }}$ & Tolerance & Variance inflation factor & Durbin-Watson \\
\hline Ambivalent attachment style & 0.41 & 0.17 & 18.16 & 0.001 & 0.04 & 0.08 & 1.37 & 0.17 & .80 & 1.24 & 1.50 \\
Avoidant attachment style & & & & & 0.12 & 0.30 & 4.94 & 0.001 & .83 & 1.20 & \\
Resiliency & & & & & -0.03 & -0.19 & -3.30 & 0.001 & .96 & 1.04 & \\
\hline
\end{tabular}

In this regression analysis, the dependent variable was obsessive-compulsive personality disorder, and the independent variables were ambivalent attachment style, avoidant attachment style, and resiliency.

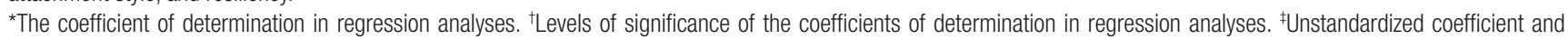

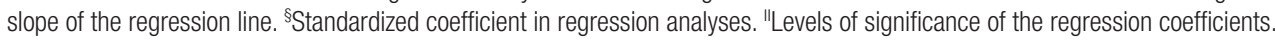

Table 4. Regression analysis of impact of attachment style on obsessive-compulsive personality disorder with modified resiliency variable

\begin{tabular}{lcccccccccccc}
\hline \multicolumn{1}{c}{ Prediction variable } & $\mathrm{R}$ & $\mathrm{R}^{2 *}$ & $\mathrm{~F}$ & $\mathrm{P}_{\text {-value }}^{\dagger}$ & $\mathrm{B}^{\ddagger}$ & $\mathrm{B}^{\S}$ & $\mathrm{t}$ & $\mathrm{P}_{\text {-value }}^{\|}$ & $\mathrm{r}$ (partial) & Tolerance & Variance inflation factor & Durbin-Watson \\
\hline (Constant) & & & & & 3.97 & & 9.58 & 0.001 & & & & \\
Ambivalent attachment style & 0.37 & 0.13 & 20.97 & 0.001 & 0.04 & 0.09 & 1.57 & 0.12 & 0.09 & .85 & 1.18 & \\
Avoidant attachment style & & & & & 0.26 & 0.32 & 5.19 & 0.001 & 0.30 & .85 & 1.18 & \\
\hline
\end{tabular}

In this in regression analyses, dependent variable was obsessive-compulsive personality disorder, independent variables were ambivalent attachment style and avoidant attachment style, and moderating variable was resiliency.

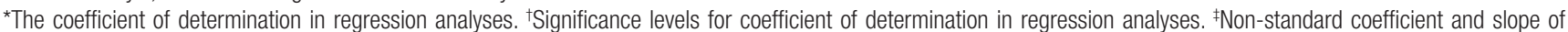
regression line. ${ }^{\S}$ Standard coefficient in regression analyses. "Significance levels for regression coefficients.

and obsessive-compulsive personality disorder. The coefficient for this effect was 0.32 , which indicated a positive effect of avoidant attachment style on obsessive-compulsive personality disorder (Table 4).

\section{DISCUSSION}

The purpose of this study was to investigate the relationships between attachment styles and resiliency with obsessive-compulsive personality disorder. People with ambivalent/avoidant attachment styles have obsessive-compulsive personality disorder more than others who do not have those attachment styles. Some potential explanations of these results are related to the suggestion that the roots of child anxiety can be discovered in the mother-child relationship. Insecure mothers are less responsive to their children compared with mothers with secure attachment styles. Insecure mothers do not give timely attention to the needs of their children and respond to the child only when they want. Therefore, these children recognize that their mothers are unreliable for meeting their needs. As a result, these children realize that the world is an insecure place for them, and they adopt a pessimistic view of others and the world around them. Therefore, people with insecure attachment styles (avoidant and ambivalent) have a greater risk for developing various diseases and disorders and psychological damage. ${ }^{19)}$ The results of several studies have indicated that insecure attachments make a person vulnerable to symptoms of anxiety and predict anxiety disorders to a great extent. ${ }^{20)}$ Overall, the results of the present study were consistent with the findings of previous studies. ${ }^{21,22)}$ People with insecure attachment style (avoidant and ambivalent) do not have feelings of peace and comfort. Therefore, they have a constant attitude that makes them unable to trust any people, entities, or events. They do not even trust themselves and are always seeking orderliness and the best, but they still do not feel secure, even when they achieve them.
Thus, people with ambivalent attachment styles feel constant anxiety, ${ }^{23)}$ and people with obsessive-compulsive personality disorder feel this same constant anxiety. ${ }^{1)}$ The results of this study can be explained by this relationship.

The results indicated the absence of a relationship between the secure attachment style and obsessive-compulsive personality disorder. Mental health disorders, such as obsessive-compulsive personality disorder, are a mental-social phenomenon that requires a good understanding and accurate interpretation of the relevant issues. Every culture views mental health according to their specific criteria. The goal of every society is to establish conditions in which all people's mental health is guaranteed. In consideration of these cultural conditions, the results of this study might be inconclusive because the questionnaire that we used in our study was based on Western culture, but our subjects were Iranians with different cultural values than those who live in Western societies.

The results of this study showed that resiliency is effective against obsessive-compulsive personality disorder. These results are supported by observations that resilient people can resist and overcome anxiety and stress factors. Therefore, resiliency leads to the adaptation and greater compatibility of people under difficult and unpleasant living conditions and vice versa: people with low resiliency are prone to anxiety disorders, such as obsessive-compulsive personality disorder, because they cannot manage stressful factors. In addition, people with high levels of resiliency have more self-confidence and effective coping strategies against toxic situations. As a result, they easily overcome stressful conditions and interpret stressful life events in a way that is tolerable for them. Enhancing resiliency can help an individual resist and overcome anxiety, stress, and other factors that cause psychological problems. ${ }^{24,25)}$ Various studies have shown that reductions in resiliency are associated with feelings of mental pressure, anxiety, and de- 
pression when facing life events. ${ }^{26)}$ Thus, resiliency moderates stress and the inability to cope with stressful situations. ${ }^{27)}$ The results of this study were consistent with the findings of Haddadi and Besharat ${ }^{28)}$ in 2010 who investigated the relationship of resiliency with indicators of vulnerability, such as psychological stress, depression, anxiety, and mental health. The resiliency results suggested that resiliency and avoidant attachment style contribute to obsessive-compulsive personality disorder and that subjects with more resiliency who do not have an avoidant attachment style develop obsessive-compulsive personality disorder less frequently. Thus, therapists should consider these two variables related to obsessive-compulsive personality disorder. When resilience is kept constant, an avoidant attachment style has a greater impact on obsessive-compulsive disorder, which suggests that individuals with more resilience are affected less by the negative effects of an avoidant attachment style and individuals with less resilience are affected more by the negative impact of the attachment style. A study titled "Attachment, Resilience and Prevention" suggested that a secure attachment style provides the necessary resilience in individuals for coping with unpleasant events so that they can cope with them as adults in the future, without psychological damage. ${ }^{29)}$ Resilience can control the negative effects of an avoidant attachment style on obsessive compulsive disorder. Therefore, it is recommended that therapists consider methods to improve the resilience of individuals with obsessive-compulsive disorder who have an avoidant attachment style. Because this study was correlational, future studies need to investigate the effects on subjects of resiliency training, which is an important component of positive psychology. Because the subjects examined in this study were students, the generalizability of these results to other populations should be performed with caution. Therefore, it is recommended that similar studies be performed in other communities and societies.

In addition to the subjects all being students, another limitation of this study was that the study was cross-sectional. Thus, future studies need to investigate the effects of attachment styles and resiliency on obsessive-compulsive personality disorder in longitudinal studies.

Please note that this study was conducted in Iran, which has its own cultural values and racial conditions. Thus, to extend the results, additional investigations are needed in other societies to determine the generalizability of the results.

\section{CONFLICT OF INTEREST}

No potential conflict of interest relevant to this article was reported.

\section{ACKNOWLEDGMENTS}

The authors wish to thank all of the people who participated in the study and the Substance Abuse Prevention Research Center, Kermanshah University of Medical Sciences, Kermanshah.

\section{REFERENCES}

1. American Psychiatry Association. The revised passage of the statistical and recognition guide of mental disorders, 2000. Tehran: Sokhan Publication; 2005.

2. Barrett PM, Healy-Farrell L, Piacentini J, March JS. Obsessive-compulsive disorder in childhood and adolescence: description and treatment. In: Barrett PM, Ollendick TH, editors. Handbook of interventions that work with children and adolescents: prevention and treatment. Hoboken (NJ): John Wiley \& Sons; 2004. p. 187-206.

3. Bowlby J. Attachment and loss: sadness and depression. New York (NY): Basic Books; 1980.

4. Besharat MA. Therapeutic bases of attachment theory. Psychol Rooyesh 2012;1:15-38.

5. Karami J, Zakiei A, Alikhani M. The role of gender differentiation and attachment styles in predicting the incidence alexithymia. Med J Tabriz Univ Med Sci Health Serv 2013;35:74-81.

6. Bowlby J. Attachment and loss. Volume 1: attachment. New York (NY): Basic Books; 1969.

7. Mikulincer M. Adult attachment style and information processing: individual differences in curiosity and cognitive closure. J Pers Soc Psychol 1997;72:1217-30.

8. McAllister M, McKinnon J. The importance of teaching and learning resilience in the health disciplines: a critical review of the literature. Nurse Educ Today 2009;29:371-9.

9. Peng L, Zhang J, Li M, Li P, Zhang Y, Zuo X, et al. Negative life events and mental health of Chinese medical students: the effect of resilience, personality and social support. Psychiatry Res 2012;196:138-41.

10. Bonanno GA. Loss, trauma, and human resilience: have we underestimated the human capacity to thrive after extremely aversive events? Am Psychol 2004;59:20-8.

11. Masten AS. Ordinary magic: resilience processes in development. Am Psychol 2001;56:227-38.

12. Connor KM, Davidson JR. Development of a new resilience scale: the Connor-Davidson Resilience Scale (CD-RISC). Depress Anxiety 2003;18:76-82.

13. Basu SR. Economic growth, well-being and governance under economic reforms evidence from Indian states. Geneva: Graduate Institute of International Studies; 2004.

14. Friborg O, Barlaug D, Martinussen M, Rosenvinge JH, Hjemdal O. Resilience in relation to personality and intelligence. Int J Methods Psychiatr Res 2005;14:29-42.

15. Hazan C, Shaver P. Romantic love conceptualized as an attachment process. J Pers Soc Psychol 1987;52:511-24.

16. Taheri M. The relationship between mothers' attachment styles and social maturation of students mentally retarded students boys Shiraz. Hakim Res J 2009; 7:18-20.

17. Jowkar B. The mediating role of resilience in the relationship between general and emotional intelligence and life satisfaction. Contemp Psychol 2007;2:3-12.

18. Brannigan G. Experiencing psychology: active learning adventures. Tehran: Amir Kabir; 2003.

19. Lenzenweger MF, Clarkin JF. Major theories of personality disorder. 2nd ed. New York (NY): Guilford Press; 2005. 
20. Kobak R, Cassidy J, Lyons-Ruth K, Ziv, Y. Attachment and developmental psychopathology. In: Cicchetti D, Cohen DJ, editors. Developmental psychopathology. New York (NY): Wiley; 2006.

21. Safford SM, Alloy LB, Crossfield AG, Morocco AM, Wang JC. The relationship of cognitive style and attachment style to depression and anxiety in young adults. J Cogn Psychother 2004;18:25-41.

22. Williams NL, Riskind JH. Adult romantic attachment and cognitive vulnerabilities to anxiety and depression: examining the interpersonal basis of vulnerability models. J Cogn Psychother 2004;18:7-24.

23. Besharat MA, Tolaeian F, Lavasani MG. Comparison of attachment styles in individuals with gender identity disorder and healthy individuals. J Forensics 2012;18:89-97.

24. Connor KM. Assessment of resilience in the aftermath of trauma. J
Clin Psychiatry 2006;67 Suppl 2:46-9.

25. Wilson JP, Drozdek B. Broken spirits: the treatment of traumatized asylum seekers, refugees, and war and torture victims. New York (NY): Brunner-Routledge; 2004.

26. Antonovsky A. Unraveling the mystery of health: how people manage stress and stay well. San Francisco (CA): Jossey-Bass; 1987.

27. Abolghasemi A, Varaniyab ST. Resilience and perceived stress: predictors of life satisfaction in the students of success and failure. Procedia Soc Behav Sci 2010;5:748-52.

28. Haddadi P, Besharat MA. Resilience, vulnerability and mental health. Procedia Soc Behav Sci 2010;5:639-42.

29. Svanberg PO. Attachment, resilience and prevention. J Ment Health 1998;7:543-78. 